\title{
TENDENCIAS Y DESARROLLO DE LAS TECNOLOGÍAS DE LA INDUSTRIA 4.0 EN EL SECTOR SALUD
}

\author{
TRENDS AND DEVELOPMENT OF INDUSTRY 4.0 TECHNOLOGIES IN \\ HEALTHCARE
}

\author{
DOI: https://doi.org/10.17981/iimsor.05.01.01
}

Review Article - Reception Date: May 18, 2020-Acceptance Date: Jun12, 2020

\author{
Laura Montesino Soraca ${ }^{1}$, Orlando Álvarez Mejia ${ }^{1}$, Alfonso R. Romero-Conrado ${ }^{1}$ \\ ${ }^{1}$ Universidad de la Costa. Barranquilla, (Colombia)
}

aromero17@cuc.edu.co

To reference this paper:

L. Montesino, O. Álvarez y A. Romero-Conrado "Tendencias y Desarrollo de las Tecnologías de la Industria 4.0 en el Sector Salud", IJMSOR, vol. 5, no. 1, pp. 1 - 6 2020. https://doi.org/10.17981/ijmsor.05.01.01

\begin{abstract}
Resumen- Este artículo presenta una revisión bibliográfica de las diferentes tendencias y desarrollo de las tecnologías de la industria 4.0 en el sector salud, la cual, se ha consolidado como una tecnología fundamental para optimizar diferentes procedimientos y consolidar procesos, profesionales y centros de salud mucho más eficientes. Así, dichas tecnologías han dado paso a una extensa variedad de productos y servicios inteligentes, reestructurando los límites del ámbito de la salud. En este sentido, el objetivo fundamental es poder identificar cuáles son las tendencias en el desarrollo e implementación de las tecnologías 4.0 en el sector de la salud. Para lograr esto, se utiliza una metodología que consiste en una revisión sistemática de diversas fuentes académicas sobre el tema en cuestión. De esta manera, se destacan distintas aplicaciones y beneficios en el sector salud en el contexto de la industria 4.0 entre las que destacan: generar nuevos métodos para la detección, prevención, tratamiento y diagnóstico de enfermedades y consolidar una mejor atención médica, más exacta y oportuna. Por último, se concluye que la industria 4.0 puede entenderse como una nueva esfera en la cual se han fomentado distintos recursos para aprovechar todo el potencial que ofrece el conjunto de innovaciones tecnológicas en el sector de la salud.
\end{abstract}

Palabras clave - Industria 4.0, Sector salud, Tecnología, Revisión.

\begin{abstract}
This article presents a bibliographic review of the different trends and development of the technologies of the 4.0 industry in the healthcare sector, which, has been consolidated as a fundamental technology to optimize different procedures and to consolidate processes, professionals and much more efficient healthcare centers. Thus, these technologies have given way to a wide variety of intelligent products and services, restructuring the limits of the health field. In this sense, the fundamental objective is to be able to identify the trends in the development and implementation of 4.0 technologies in the health sector. To achieve this, it uses a methodology consisting of a systematic review of various academic sources on the subject in question. Therefore, it highlights different applications and benefits in the health sector in the context of the 4.0 industry, among which are: generating new methods for the detection, prevention, treatment and diagnosis of diseases and consolidating better, more accurate and timely medical care. Finally, it is concluded that industry 4.0 can be understood as a new area in which different resources have been promoted to take advantage of the full potential offered by the set of technological innovations in the health sector.
\end{abstract}

Key Words - Industry 4.0, Health sector, Technology, Review. 


\section{INTRODUCCIÓN}

A lo largo del tiempo las industrias deben de ir en pro de evolucionar para así estar a la vanguardia y no estancarse. La industria 4.0 es un término que nace como una estrategia idealizada en el desarrollo de la tecnología en el sector industrial para potencializar las falencias a través de la disminución de costos y agilización de los procesos internos sin olvidar la alta calidad de estos mismos. Tal como lo indican [1]-[3] este suceso representa uno de los avances humanos dirigidos a beneficiar a más de un sector de la sociedad, así, es casi que indudable que la atención médica, se beneficie significativamente de la implementación e integración de conceptos y tecnologías de la industria 4.0. La tecnología es el factor fundamental y clave para que se logre obtener el procesamiento de los datos de manera eficaz y contribuyendo así con la disminución del porcentaje de error de procesos, así lograr la eficiencia en el sistema implementado en el sector salud. Así, de acuerdo a [4] este fenómeno, también conocido como la cuarta revolución industrial, combina los espacios físicos, digitales y biológicos, para así cambiar la industria de la salud desde todos los ámbitos de la misma.

La industria 4.0 logró generar un vuelco positivo en el sector de la medicina, el cual es uno de los más grandes y vitales para la socioeconomía, y, a su vez es también uno de los sectores más complejos. En este ámbito, cada vez se ve necesario más estudios investigativos, más innovaciones tecnológicas, y una mayor precisión, financiación e implementación de nuevos proyectos enfocados en la salud y en el bienestar de las personas. De esta manera, [5], indica que son precisamente este tipo de avances tecnológicos que propone la industria 4.0, los que están dando lugar a una gran variedad de productos y servicios inteligentes, reestructurando los límites de la industria de la salud y estableciendo industrias completamente nuevas. En este sentido, el objetivo fundamental de esta revisión es poder identificar cuáles son las tendencias en el desarrollo e implementación de las tecnologías en la industria 4.0 en el sector de la salud. Así, este artículo está organizado de la siguiente forma: la sección 2 muestra los aspectos metodológicos bajo los cuales se desarrolló la revisión sistemática; posteriormente, la sección 3 expone los resultados de la investigación; y, finalmente, en la sección 4 se plantean las conclusiones sobre los hallazgos encontrados.

\section{METODOLOGÍA}

\subsection{Diseño:}

Se realizó una revisión sistemática de documentos referentes al desarrollo de tendencias, herramientas y aplicaciones de la industria 4.0 en el sector de la salud a nivel mundial. Esta revisión es acorde con la pregunta de investigación, que es ¿Cuáles son las tendencias en el desarrollo e implementación de las tecnologías en la industria 4.0 ? en el sector de la salud a nivel mundial? Además de que se realizaron filtros de búsqueda como los años en los que se publicaron los artículos, que comprendió desde 2018-2021 y que solo fueran artículos y artículos de revisión para obtener resultados más recientes.

\subsection{Estrategia de búsqueda}

Para la búsqueda de estudios originales se consultó la base de datos Scopus, mediante la siguiente ecuación de búsqueda: ( TITLE-ABS-KEY ( industry 4.0 ) AND TITLE-ABS-KEY ( health) ) AND DOCTYPE ( ar OR re ) AND ACCESSTYPE ( OA) AND PUBYEAR > 2018 AND PUBYEAR $<2021$

Las palabras claves fueron: Industry 4.0, Health, Health 4.0 .

\section{Criterios de inclusión y exclusión}

Los criterios de inclusión utilizados dentro de la revisión fueron:

1. El artículo expone una aplicación de alguna de las tecnologías de la industria 4.0 en el sector salud a nivel mundial

2. El artículo expone o aporta a los conocimientos del desarrollo de la industria 4.0 en el sector salud a nivel mundial.

El criterio de exclusión fue:

1. Se excluyeron artículos que exponían la utilización de algunas de las tecnologías de la industria 4.0 en un sector diferente a la salud o no proporcionaban la suficiente información para identificar su aplicación en el sector.

\subsection{Extracción de datos}

Durante la búsqueda inicial se encontraron 61 estudios, después de realizar el respectivo análisis de los objetivos de los estudios se escogieron 20. Los artículos escogidos respondían de alguna manera con la pregunta de investigación que es ¿Cuáles son las tendencias en el desarrollo e implementación de las tecnologías en la industria 4.0 en el sector de la salud a nivel mundial? Finalmente 20 artículos se escogieron para su revisión final. 


\section{RESULTADOS}

\subsection{Enfoque}

En la revisión literaria de los documentos, se logró apreciar que estaban enfocados fundamentalmente al uso de la industria 4.0 en el sector de la salud, teniendo distintos objetivos entre los que destacan: la prevención, detección, tratamiento y diagnóstico de enfermedades, la creación de implantes para las personas, el cuidado de los datos de los pacientes, la contaminación del ambiente, seguridad en el trabajo, buscar una posible cura y para el virus COVID19, que es en estos momentos un problema para el sistema de salud a nivel mundial. Así, estos diferentes textos, proponen maneras para ayudar o contribuir en el mejoramiento de la prestación de salud, para no solo optimizar la calidad de los servicios sino también, evitar un colapso total del sistema, problema que generaría una emergencia sanitaria.

El sector de la salud ha estado tomando importancia en los últimos años, es por esto por lo que la industria 4.0 juega un papel importante para el mejoramiento de esta, permitiendo que se le pueda brindar a las personas una mejor atención, y de esta manera garantizar un bienestar, un mejor vivir y tratar las enfermedades de la manera óptima, rápida y oportuna posible, evitando que el paciente sufra de deterioro o que se pueda detectar a tiempo.

\subsection{Aplicaciones}

Tabla 1. Aplicaciones y tecnologías de la industria 4.0 en el sector de la salud en los últimos 5 años. Elaboración propia.

\begin{tabular}{|l|l|}
\hline Tecnologías & \multicolumn{1}{|c|}{ Aplicación } \\
\hline $\begin{array}{l}\text { Internet de le programación de tareas; } \\
\text { las cosas }\end{array}$ & $\begin{array}{l}\text { Mejorar la } \\
\text { maximizar la utilización de los recursos; } \\
\text { acceder a los datos de almacenamiento } \\
\text { del paciente de manera eficiente; } \\
\text { prevenir enfermedades. }\end{array}$ \\
\hline $\begin{array}{l}\text { Fábricas } \\
\text { inteligentes }\end{array}$ & $\begin{array}{l}\text { Optimizar actividades en los centros } \\
\text { médicos; recopilar información de los } \\
\text { pacientes; mejorar la accesibilidad de } \\
\text { datos entre proveedores de atención } \\
\text { médica. }\end{array}$ \\
\hline ANN & $\begin{array}{l}\text { Mejorar el diagnóstico y tratamiento de } \\
\text { pacientes. }\end{array}$ \\
\hline $\begin{array}{l}\text { Sistemas de } \\
\text { información } \\
\text { sanitaria }\end{array}$ & $\begin{array}{l}\text { Facilitar el intercambio de datos de } \\
\text { salud de las personas; mejorar la } \\
\text { atención de los pacientes en diferentes } \\
\text { centros de salud. }\end{array}$ \\
\hline $\begin{array}{l}\text { Inteligencia } \\
\text { artificial }\end{array}$ & $\begin{array}{l}\text { Detectar, analizar y diagnosticar } \\
\text { enfermedades; obtener servicios más } \\
\text { rápidos y de mejor calidad; fabricar } \\
\text { implantes y llevar a cabo cirugías según } \\
\text { las necesidades de cada paciente; reducir } \\
\text { los riesgos para la salud de los usuarios } \\
\text { y de los trabajadores médicos. }\end{array}$ \\
\hline
\end{tabular}

\begin{tabular}{|c|c|}
\hline Big Data & $\begin{array}{l}\text { Recopilar información de los pacientes; } \\
\text { mejorar la accesibilidad de datos entre } \\
\text { proveedores de atención médica; ayudar } \\
\text { en la prevención de enfermedades }\end{array}$ \\
\hline WASPLab ${ }^{\circledR}$ & $\begin{array}{l}\text { Detectar el crecimiento bacteriano; } \\
\text { identificar infecciones bacterianas; } \\
\text { establecer procesos en busca de la } \\
\text { preservación de la salud humana. }\end{array}$ \\
\hline $\begin{array}{l}\text { Realidad } \\
\text { aumentada } \quad y \\
\text { virtual }\end{array}$ & $\begin{array}{l}\text { Desarrollar tanto de profesionales de la } \\
\text { salud como centros de servicio mucho } \\
\text { más óptimos y eficientes; ofrecer un } \\
\text { mejor servicio enfocado en las } \\
\text { necesidades particulares de cada } \\
\text { paciente. }\end{array}$ \\
\hline $\begin{array}{l}\text { Computación } \\
\text { en la nube }\end{array}$ & $\begin{array}{l}\text { Optimizar la atención médica; } \\
\text { intercambiar datos médicos que permita } \\
\text { el acceso y colaboración entre varias } \\
\text { entidades prestadoras del servicio de } \\
\text { salud. }\end{array}$ \\
\hline
\end{tabular}

La industria 4.0 está siendo empleada para múltiples usos relacionados con el área de la salud, buscando generar nuevos métodos para la detección, prevención, tratamiento y diagnóstico de enfermedades, garantizando a las personas una mejor calidad de vida, bienestar, una mejor atención médica, más exacta y oportuna que le permita a los pacientes actuar de manera rápida y efectiva.

De esta manera, tal y como describen [6], la adopción de tecnologías de la industria 4.0 como el paradigma de la computación en la nube y el sistema del Internet de las cosas, ha permitido brindar nuevas herramientas para optimizar la atención médica. En el artículo, los autores proponen un modelo para optimizar la selección de máquinas virtuales con el fin de mejorar el rendimiento de los sistemas de salud. Este modelo, busca enriquecer la programación de tareas al reducir el tiempo de ejecución de solicitudes de los usuarios, maximizar la utilización de los recursos facilitando el almacenamiento de datos y proporcionar un mecanismo de recuperación de información eficiente para todos los beneficiarios del sistema de salud.

En el mismo sentido, [7] en su artículo "Development of soft computing tools and IoT for improving the performance assessment of analysers in a clinical laboratory" basándose en el sistema de Internet de las cosas, desarrollaron un modelo para realizar la evaluación de rendimiento del equipo sanitario, en el que no solo se redujo el tiempo que le toma a un usuario realizar el proceso, sino que también se logró que fuera más eficiente al momento de obtener los resultados de la prueba. Además, en este modelo se utilizó una herramienta de predicción de fallas con el uso de inteligencia artificial, todo con el propósito de mejorar la gestión de calidad tanto de los empleados de un centro de salud, como de toda la institución en general. De igual forma, [8] en el contexto de la Industria 4.0 y con el propósito de facilitar el 
intercambio de datos de salud de las personas - dirigido con el objetivo de mejorar la atención de los pacientes en diferentes centros de salud-, plantea la implementación de aplicaciones de sistemas de información sanitaria a partir de la computación en la nube.

Igualmente, en el artículo "Fourth industrial revolution for development: The relevance of the cloud federation in health support" [9] a partir de la utilización del sistema de la nube, proponen un sistema de intercambio de datos médicos que permita el acceso y colaboración entre varias entidades prestadoras del servicio de salud. Esto, con el fin de maximizar la utilización de recursos y facilitar la ejecución de tareas tales como la atención médica y diferentes procedimientos presenciales. En el mismo orden de ideas, [10] principalmente a través de la tecnología "Blockchain" y junto con otras como la de Big Data, propone un modelo para la optimización de actividades en los centros médicos tales como el diagnóstico de enfermedades, recopilar información de los pacientes y mejorar la accesibilidad de datos entre proveedores de atención médica.

Por otro lado, [11] manifiestan el uso del sistema de Internet de las cosas, y esta vez junto con los sistemas ciber-físicos, para mejorar la eficiencia en la prestación de servicios de salud. Estos autores, indican que lo ideal sería lograr que el sistema de salud pueda operar como una "fábrica inteligente", donde se monitoreen constantemente los procesos físicos y se virtualicen los demás procedimientos para garantizar la plena eficiencia. De esta manera, el artículo expone que la implementación de dichos procesos permitirá optimizar actividades como la interacción entre los usuarios y los trabajadores, la asistencia por parte del sector administrativo, y especialmente, la distribución de información sobre prevención de enfermedades y el diagnóstico de los pacientes.

De igual forma, [4] en el artículo "Fourth Industrial Revolution: An Impact on Health Care Industry" proponen la detección, análisis y diagnóstico de enfermedades a partir de técnicas de inteligencia artificial, enfocándose especialmente en la obtención de servicios más rápidos y de calidad en áreas remotas. Además, indican que el uso de la nanotecnología, especialmente la inspirada en el sistema de Redes neuronales artificiales o ANN por sus siglas en inglés, optimiza el diagnóstico y tratamiento de pacientes. De manera similar, también, [12] plantean el empleo de herramientas como la Big Data para ayudar en la prevención de enfermedades, particularmente de tipo infecciosas. Esta idea, se desarrolla a partir de un esquema para monitorear las enfermedades con el uso de las grandes compilaciones de datos. Además, los autores plantean que con esta tecnología se optimizará la obtención de datos personales de los pacientes, reduciendo los errores por parte de los centros de salud al otorgar medicamentos.

En otro sentido, autores tales como [13], [14] publicaron artículos enfocados en la comprensión de manera holística de los beneficios que trae la implementación de las tecnologías de la Industria 4.0 en el sector salud. Así, mientras que [14] destacan instrumentos como tarjetas inteligentes, sistemas de telemedicina y de información compartida, [13] ejemplifican beneficios que varían desde la implementación de equipos con sensores, robots, e impresoras tridimensionales en los centros físicos de salud, hasta la utilización de sistemas de información para optimizar la entrega de información y la atención médica. De forma que, en ambos artículos se resalta el papel que tienen dichas tecnologías en las nuevas formas de proporcionar servicios y productos de salud. En este orden de ideas, -y en relación con la aplicación específica de la industria 4.0- con el artículo titulado "Reconfigurable measuring system for the automatic detection of bacterial growth in a specimen processing platform", [15] describen un sistema de medición impulsado por la tecnología de procesamiento de WASP Lab®, para detectar el crecimiento bacteriano y particularmente identificar infecciones bacterianas, todo con el objetivo de establecer procesos en busca de la preservación de la salud humana. De igual manera, otro estudio específico es el propuesto por [16], quienes a partir del sistema de Internet de las cosas, herramientas de inteligencia artificial y fábricas inteligentes, proponen modelos de fabricación de implantes y dispositivos ortopédicos. En este artículo, los autores enfocados en el mejoramiento de conservación de la salud, indican diferentes fórmulas para fabricar implantes y llevar a cabo cirugías según las necesidades de cada paciente, minimizando los riesgos de tales procedimientos. Igualmente, en "Industry 4.0 technologies and their applications in fighting COVID-19 pandemic" [17] presentan estrategias y propuestas desde tecnologías de la industria 4.0, como la telemedicina y sistemas de información digital, para la prevención y tratamiento de casos de COVID-19. Al mismo tiempo, también con instrumentos de inteligencia artificial y la computación en la nube, los autores buscan reducir los riesgos para la salud de los usuarios y de los trabajadores médicos.

Desde otra perspectiva, con el objetivo de detectar agentes contaminantes en el ambiente a partir del sistema de Internet de las cosas, [18] proponen la importancia de contar con un sistema capaz de identificar el nivel de contaminación del aire en los centros de salud. Así, argumentan que tal contaminación afecta significativamente el entorno de las personas, y dicho sistema mejorará el desempeño laboral, la salud y el bienestar de las personas. De igual forma, en el artículo titulado "Occupational health and work safety systems in compliance with industry 4.0: Research directions" escrito por [19] se realiza una revisión literaria en donde se analizan diferentes propuestas para mejorar y promover el cuidado de la salud y la seguridad en el trabajo, a partir del uso de herramientas de inteligencia artificial y del sistema de internet de las cosas.

Desde otro enfoque, [20] en su artículo "Bioethics of Things: On the algorithmization of moral deliberation in clinical practice", propone una estructura para optimizar la digitalización de datos, de una manera socialmente responsable y con una perspectiva moralmente aceptable. De esta forma, mediante el análisis de opiniones y 
comportamientos de los usuarios analizados con instrumentos de inteligencia artificial y de Big Data, se pretende establecer qué se considera moralmente válido para los usuarios. Asimismo, en [21]-[23] proponen una serie de iniciativas desde el contexto de la industria 4.0 para incorporar acciones de protección tanto para la salud de los beneficiarios de los centros de salud, como para mitigar los daños al entorno medioambiental debido a los procesos que se vayan a llevar a cabo. Así, relaciona directamente el concepto de responsabilidad social corporativa del sector salud con el de las prácticas sostenibles, para entonces, lograr implementar tecnologías en pro de mejorar la calidad de servicio que se les ofrece a los pacientes.

Por otro lado, [24] en su artículo titulado "Development of smart nursing homes using systems engineering methodologies in industry 4.0", mediante un proceso de desarrollo interactivo y de ciclo de vida basado en el modelo Vee de ingeniería de sistemas, proponen ciertas herramientas para asignar, refinar y optimizar el servicio de centros de salud -cabe resaltar que en el texto, indican el ejemplo con un hogar de ancianos-. Así, estudian e indican los requisitos para un desarrollo sistemático de centros de cuidado de personas mayores a partir de la implementación y sincronización con un sistema ciberfísico; buscando fundamentalmente proponer un sistema holístico que tenga en cuenta las necesidades particulares de sus beneficiarios. De la misma manera, [25] a partir de instrumentos como el sistema de Internet de las cosas, proponen un nuevo paradigma para mejorar los servicios digitales de salud y atención para los pacientes. En este mismo sentido, [26] manifiesta nuevos modelos de atención médica integrando tecnologías propias de la industria 4.0 para ofrecer servicios de salud, e igualmente centros de salud, de manera más eficiente. Así, los autores mencionan la implementación de herramientas como los sistemas ciber-físicos, el sistema de Internet de las cosas y aplicaciones de salud móvil conocidas como "mHealth".

\section{CONCLUSIONES}

Esta revisión ha permitido evidenciar la necesidad de examinar y analizar las tendencias y el desarrollo de las distintas tecnologías de la industria 4.0 en el sector de la salud. Así, a partir de la literatura revisada en este artículo, se dio a conocer los múltiples beneficios de la implementación de las diferentes herramientas tecnológicas tales como: mejorar la programación de tareas, maximizar la utilización de los recursos, facilitar el acceso a los datos de almacenamiento de los pacientes, la reducción de costos y riesgos por parte del sector sanitario, optimizar la prevención de enfermedades, crear modelos eficientes para el diagnóstico de enfermedades, mejorar la calidad en los sistemas de atención médica, consolidar sistemas de información compartida, asegurar la seguridad en el entorno de los centros de salud tanto para pacientes como trabajadores, entre muchos otros. Asimismo, los resultados de la investigación permiten concluir que el concepto de la industria 4.0 puede entenderse como un nuevo ámbito en el cual se han fomentado distintos recursos, que varían desde el tiempo invertido, el capital humano, los medios financieros, hasta las diferentes creaciones tanto físicas como digitales; para aprovechar todo el potencial que ofrece el conjunto de innovaciones tecnológicas en el sector de la salud. De esta forma, los servicios innovadores propuestos desde la industria 4.0 como el uso de chips electrónicos, dispositivos médicos inteligentes, datos electrónicos y sistemas de realidad virtual, han contribuido al desarrollo tanto de profesionales de la salud como de los mismos centros de servicio mucho óptimos y eficientes, enfocados en atender las necesidades particulares de los pacientes y ofrecer un mejor servicio.

\section{AGRADECIMIENTOS}

Este artículo fue elaborado en el marco del Diplomado en Producción Investigativa en Desarrollo Sostenible de la Universidad de la Costa. Los autores agradecen la colaboración y asesoría de la profesora Karen Elena Salas Viloria, coordinadora del Laboratorio Virtual en Producción Científica; y del ingeniero Henry Maury Ardila, Vicerrector de Ciencia, Tecnología e Innovación.

\section{REFERENCIAS}

[1] H. Lasi, P. Fettke, H.-G. Kemper, T. Feld, and M. Hoffmann, "Industrie 4.0 Auslöser," Wirtschaftsinformatik, vol. 56, no. 4, pp. 261-264, 2014.

[2] A. R. Romero-Conrado, L. J. Castro-Bolaño, J. R. Montoya-Torres, and M. Á. Jiménez Barros, "Operations research as a decision-making tool in the health sector: A state of the art," DYNA, vol. 84, no. 201, p. 129, May 2017.

[3] L. Galvis Leal, L. Orozco De Alba, and A. R. RomeroConrado, "Desarrollo, tendencias, aplicaciones y herramientas de la industria 4.0 en el sector textil," Boletín Innovación, Logística y Operaciones - BILO, vol. 2, no. 1, Aug. 2020.

[4] P. Jayanthi, M. Iyyanki, A. Mothkuri, and P. Vadakattu, "Fourth Industrial Revolution: An Impact on Health Care Industry," in Advances in Intelligent Systems and Computing, 2020, vol. 965, pp. 58-69.

[5] H. A. Park, "Are We Ready for the Fourth Industrial Revolution?," Yearb. Med. Inform., 2016.

[6] M. Elhoseny, A. Abdelaziz, A. S. Salama, A. M. Riad, K. Muhammad, and A. K. Sangaiah, "A hybrid model of Internet of Things and cloud computing to manage big data in health services applications," Futur. Gener. Comput. Syst., vol. 86, pp. 1383-1394, Sep. 2018.

[7] M. S. Packianather, N. L. Munizaga, S. Zouwail, and M. Saunders, "Development of soft computing tools and IoT for improving the performance assessment of analysers in a clinical laboratory," in 2019 14th Annual Conference System of Systems Engineering, SoSE 2019, 2019, pp. 158-163.

[8] X. Larrucea, M. Moffie, S. Asaf, and I. Santamaria, "Towards a GDPR compliant way to secure European cross border Healthcare Industry 4.0," Comput. Stand. Interfaces, vol. 69, p. 103408, Mar. 2020.

[9] O. O. Ajayi, A. B. Bagula, and K. Ma, "Fourth industrial revolution for development: The relevance 
of cloud federation in healthcare support," IEEE Access, 2019.

[10] S. Tanwar, K. Parekh, and R. Evans, "Blockchainbased electronic healthcare record system for healthcare 4.0 applications," J. Inf. Secur. Appl., 2020.

[11] M. Alloghani, D. Al-Jumeily, A. Hussain, A. J. Aljaaf, J. Mustafina, and E. Petrov, "Healthcare Services Innovations Based on the State of the Art Technology Trend Industry 4.0," in Proceedings - International Conference on Developments in eSystems Engineering, DeSE, 2019, vol. 2018-Septe, pp. 64-70.

[12] D. Sudana and A. W. R. Emanuel, "How Big Data in Health 4.0 Helps Prevent the Spread of Tuberculosis," in Proceedings - 2019 2nd International Conference on Bioinformatics, Biotechnology and Biomedical Engineering. Bioinformatics and Biomedical Engineering, BioMIC 2019, 2019.

[13] W. Z. Khan, M. H. Rehman, H. M. Zangoti, M. K. Afzal, N. Armi, and K. Salah, "Industrial internet of things: Recent advances, enabling technologies and open challenges," Comput. Electr. Eng., 2020.

[14] G. Aceto, V. Persico, and A. Pescapé, "Industry 4.0 and Health: Internet of Things, Big Data, and Cloud Computing for Healthcare 4.0," Journal of Industrial Information Integration, vol. 18. Elsevier B.V., p. 100129, Jun-2020.

[15] P. Bellitti, M. Bona, M. Borghetti, E. Sardini, M. Serpelloni, and S. Fontana, "Reconfigurable measuring system for the automatic detection of bacterial growth in a specimen processing platform," Acta IMEKO, 2019.

[16] M. Javaid and A. Haleem, "Impact of industry 4.0 to create advancements in orthopaedics," J. Clin. Orthop. Trauma, 2020.

[17] M. Javaid, A. Haleem, R. Vaishya, S. Bahl, R. Suman, and A. Vaish, "Industry 4.0 technologies and their applications in fighting COVID-19 pandemic," Diabetes Metab. Syndr. Clin. Res. Rev., 2020.

[18] S. Sun, X. Zheng, J. Villalba-Díez, and J. Ordieres-
Meré, "Indoor air-quality data-monitoring system: Long-term monitoring benefits," Sensors (Switzerland), vol. 19, no. 19, p. 4157, Oct. 2019.

[19] M. Erol, "Occupational health and work safety systems in compliance with industry 4.0: Research directions," Int. J. Ebus. eGovernment Stud., vol. 11, no. 2, pp. 121-133, 2019.

[20] P. Calvo, "Bioethics of Things: On the algorithmization of moral deliberation in clinical practice," Filos. Unisinos, vol. 20, no. 2, pp. 155-162, 2019.

[21] G. Daú, A. Scavarda, L. F. Scavarda, and V. J. T. Portugal, "The healthcare sustainable supply chain 4.0: The circular economy transition conceptual framework with the corporate social responsibility mirror," Sustain., 2019.

[22] D. C. Landinez-Lamadrid, D. G. Ramirez-Ríos, D. Neira Rodado, K. Parra Negrete, and J. P. Combita Niño, "Shapley Value: its algorithms and application to supply chains," INGE CUC, vol. 13, no. 1, pp. 6169, Jan. 2017.

[23] A. Sánchez Comas, A. Troncoso Palacio, S. Troncoso Mendoza, and D. Neira Rodado, "Application of taguchi experimental design for identication of factors influence over $3 \mathrm{D}$ printing time with fused deposition modeling," IJMSOR, vol. 1, no. 1, pp. 4348, 2016.

[24] F. Cui, L. Ma, G. Hou, Z. Pang, Y. Hou, and L. Li, "Development of smart nursing homes using systems engineering methodologies in industry 4.0," Enterp. Inf. Syst., vol. 14, no. 4, pp. 463-479, 2020.

[25] C. Chute and T. French, "Introducing care 4.0: An integrated care paradigm built on industry 4.0 capabilities," Int. J. Environ. Res. Public Health, 2019.

[26] D. V. Dimitrov, "Medical internet of things and big data in healthcare," Healthcare Informatics Research. 2016. 